\title{
B.C.G. AND IMMUNITY
}

F. R. G. Heaf, M.D., F.R.C.P.

When a normal body is infected by tubercle bacilli, the tissues at the site of invasion immediately respond in a manner adopted towards any foreign body. There is a slight inflammatory reaction at the site of invasion. The bacilli are confronted at first with little resistance and therefore multiply rapidly and are carried by the lymphatics to the nearest

definite and after two weeks there is an increasing effort to suppress the multiplication of the bacilli. Phagocytosis becomes more active and an increase in the number of monocytes and grouping of endothelial cells is evident. At the same time there is an attempt to restrict the wanderings of the bacilli and localize the lesion. If the invading bacilli are in great numbers, or of sufficient virulence, this resistance of the tissues will be only partially successful and a further dissemination of the organisms to other tissues will take place, but the effort upon the part of the host to localize the lesion will continue wherever the bacilli may lodge. If, on the other hand, the number of bacilli is small, or the virulence is low, the complete isolation of the majority may take place along with the killing off of those that have escaped to other tissues. Most of the bacilli imprisoned in phagocytes will die and the increase in fibroblasts will tend to limit the lesion, although necrosis and caseation will be seen within the fibrous encirclement.

The power to produce this sensitivity to tubercle bacilli or tuberculo-protein is not possessed only by the living organism. Some degree of tuberculin sensitivity can be produced by phenol-killed tubercle bacilli, but it is slight and transitory. Dubos (1953)(1) has recently shown that such bacilli, when injected into mice, confer a certain degree of immunity as measured by the number of bacilli in the lungs following an injection of virulent tubercle bacilli. In mice, the degree of immunity produced by injecting non-heatkilled bacilli was similar to that following very small injections of virulent living bacilli. If immunity is measured by the speed of multiplication of the bacillus in the tissues, then non-heat-killed bacilli, attenuated and virulent ones can be said to produce similar degrees of immunity if the dose is regulated in each case. The attenuated organism is the one most satisfactory to use for this purpose and the strain prepared by Calmette and Guérin and known as B.C.G. is the one most frequently used, as it is harmless to man.

Suspensions of B.C.G. injected intradermally, or given by mouth, cause tuberculin sensitivity to develop in the vast majority 
of individuals within six weeks. In infants it may take longer. A few individuals fail to respond to the injection and remain persistently insensitive to tuberculin. It is not easy to determine the duration of the tuberculin sensitivity produced, as any natural infection by tubercle bacilli following the injection will boost up the sensitivity. So for accurate observations on the point, complete protection from invasion by virulent tubercle bacilli is necessary. When this is made it is found that some individuals lose their sensitivity within twelve months of being B.C.G. vaccinated; others maintain it for as long as five or even seven years.

Although knowledge of the duration of tuberculin sensitivity following vaccination is of considerable value, a matter of greater importance is the degree of immunity against tuberculosis conferred by the vaccine. This can be measured by noting the incidence of the disease in vaccinated and unvaccinated comparable groups who are exposed to the same risk. This is very difficult to do and it is doubtful if a controlled trial can be organised that would be free of criticism when dealing with such a multifactorial disease like tuberculosis. Trials have been made and some are still proceeding by which it is hoped to assess the value of B.C.G. vaccination. Another method of assessing the degree of immunity conferred by the vaccine has been used by Dubos (I953)(2)(3) who measured the number of bacilli in the lungs of B.C.G. vaccinated mice two weeks after they had been given injections of virulent tubercle bacilli. It was found that in the unvaccinated controls the number was 200 times as great as in the vaccinated animals. Although there is at present no scientific proof that B.C.G. vaccination confers on man immunity against contracting tuberculosis, there is strong presumptive evidence from observations that have been made in different parts of the world. That the protection afforded by B.C.G. vaccination against tuberculosis is only partial is easily appreciated by noting that persons who have been vaccinated do sometimes develop active lesions, even within a year or two of vaccination. These cases can be found in any country where B.C.G. vaccination is practised.

Among the numerous papers that have been published on the subject those of Hertzberg (I949) (4) Wallgren (I945)(5) Ferguson $\left(^{(1946)}\right)^{(6)}$ Aronson and Palmer $\left(\right.$ I946) ${ }^{(7)}$ Wilson (I947) ${ }^{(8)}$ Myers $\left(\right.$ I952) ${ }^{(9)}$ Levine $($ I952)(10) Irvine (I949)(11) van Deinse (I95I)(12) Holm (I946)(13) and Palmer (I952)(14) are all of great value and should be studied. Some recent observations by Dahlstrom and Difs (I95I)(15) are worthy of particular mention. They found that the incidence of primary tuberculosis in over 60,000 conscripts to the Swedish Army was about five times as high in the unvaccinated 
as the vaccinated and that the incidence of post primary lesions was about three times.

The results of large scale tuberculin testing and B.C.G. vaccination are given in W.H.O. Reports $(1950)^{(16)}$ from which it is claimed that no harm has been recorded from the vaccination of over eight million persons.

A perusal of the literature leads one to conclude that the risk of developing active tuberculosis from the primary infection is reduced approximately four times by B.C.G. vaccination. There is not such good evidence that the vaccine has any protective influence against post primary infections, although some authorities are of the opinion that the lesions developing in vaccinated persons are not so severe as those in the non vaccinated. There are so many factors that control the development and progress of tuberculous lesions in man that it is difficult to assess the influence of the vaccine on post primary infection. There is, however, reason to presume that the vaccine offers considerable protection against the primary infection. Good evidence of this is found in the population on the Island of Bornholm, 49.6 per cent of whom had been B.C.G. vaccinated up to the end of 1950. As a result of increased vaccination it is claimed that tuberculosis has definitely receded, but at the same time reversion from positive tuberculin reaction to a negative reaction has increased, both in those naturally infected and the B.C.G. vaccinated. Bovine tuberculosis was eradicated from the Island in 1932. In order, therefore, to keep tuberculosis in check it will be necessary to reduce natural infection to a minimum and to maintain resistance by B.C.G. vaccination, as new cases of tuberculosis and deaths from the disease " are most frequent among the negative reactors, less frequently among the positive reactors due to natural infection and least among the B.C.G. vaccinated." (17)

Although the Bornholm evidence is of great value, we must not assume that vaccinated individuals can withstand frequent large doses of virulent bacilli. It is therefore still risky to place a vaccinated child in a heavily infected tuberculous environment. It may be able to withstand small doses of virulent tubercle bacilli, but large or even continuous small infections will eventually overcome the native and acquired resistance following vaccination and active disease will develop. It must be borne in mind that usually the only significant change that can be observed following B.C.G. vaccination is that the individual becomes tuberculin positive and nobody would agree that merely the possession of tuberculin sensitivity warrants the assumption that the individual will not contract tuberculosis.

In practice we accept the criterion that a person has been 
successfully vaccinated if tuberculin sensitivity developes. This is interpreted to mean that some degree of immunity has developed. The co-relation between the degree of tuberculin sensitivity and the standard of immunity is difficult to define. It is quite certain that tuberculin sensitivity and immunity are different entities, although there is a relationship between them. It may be stated that a tuberculin positive person has a certain resistance against the infection, but a tuberculin negative reaction does not indicate an absence of resistance. In defining tuberculin sensitivity it is necessary to be clear on the strength of the tuberculin used in testing. A person of low sensitivity will be negative to high dilutions of tuberculin, but positive to low dilutions and sensitivity may be so low that it can only be detected by using B.C.G. vaccine and obtaining a mild Koch's phenomenon. All these cases can be classed as tuberculin positive but they will have vastly different degrees of tuberculin sensitivity. May we therefore ask what degree of tuberculin sensitivity must be produced by vaccination to indicate that the operation has been successful and an adequate standard of resistance has been acquired? The line is an arbitrary one and opinions vary. The World Health Organization has laid it down that all persons who do not react to 5 International Tuberculin Units (i.e. I-2000 Old Tuberculin Mantoux test) are eligible for vaccination and if this produces a positive reaction to the same strength of tuberculin, the vaccination has been successful. The Medical Research Council require Ioo T.U. for both the pre and post vaccination tuberculin tests; other authorities are content with Io T.U. It will be seen that a good deal of confusion exists with regard to the definition of a positive tuberculin reaction. It is highly probable that the most satisfactory and practical standard to work to is Io International Tuberculin Units.

In a young person it is usual that a low tuberculin sensitivity indicates a poor degree of resistance, but this is not true in the older age groups, particularly in cases complicated by pneumoconiosis or chronic respiratory infections. In these cases a high degree of resistance to tuberculosis can exist with a low skin sensitivity to tuberculin. Conversely the highly sensitive person to tuberculin may suffer from serious constitutional symptoms due to an allergic reaction to the infection, the severity of which will depend on the extent of dissemination of the bacilli through the body. There would therefore appear to be an optimum sensitivity that varies with age, and that in young persons it is not good to be either too hypersensitive or too hyposensitive. It is probable that a reaction of 5 to $8 \mathrm{~mm}$. induration following an intradermal injection of IO I.T.U. is the most satisfactory state of sensitivity. To maintain over a 
number of years this degree of tuberculin sensitivity after B.C.G. vaccination it will be necessary for some individuals to receive periodic boosting doses of either B.C.G., or occasional small doses of virulent bacilli. If these are not received, the post vaccination sensitivity will wane and eventually disappear, although if this occurs it does not necessarily mean that resistance to tuberculous infection has also disappeared, but usually it will not be so high as in individuals in which the sensitivity has been maintained.

This generalization has its exceptions. Patients suffering from sarcoidosis are frequently tuberculin negative and their serum has the power of neutralising the ability of Old Tuberculin to produce a reaction in known positive persons. (18) As would be expected, when persons with sarcoid type of lesions develop active tuberculosis, they become sensitive to tuberculin. ${ }^{(19)}$ It must be borne in mind that the tuberculin test is a measure of skin sensitivity and although antibody formation may take place centrally, the investigation is a local one and assumes that the sensitivity of all tissues is reflected in the reaction of the skin. A considerable amount of work has been done to attempt to determine to what extent the skin is the site of the sensitization process, but there are still many points on which there is disagreement. (20)

It is not surprising, therefore, that much interest has centred around the local reaction to the B.C.G. injection and it has been suggested that as about 98 per cent of those showing a local reaction at the site of vaccination are positive to Io T.U. six weeks after, it should be possible to dispense with the post vaccination test and rely on measurement of the local B.C.G. lesion. This raises the relationship between the local B.C.G. lesion and the post vaccination tuberculin reaction.

Hertzberg (1949) (21) is of the opinion that the greater the local reaction to B.C.G. vaccination, the higher is the frequency of conversion to tuberculin positivity and also the more durable state of sensitivity.

The relationship between the tuberculin sensitivity following B.C.G. vaccination and the local B.C.G. lesion is not simple and Edwards and Palmer (1953) (22) are of the opinion that "there is only a slight tendency for large B.C.G. lesions to be followed by large tuberculin reactions and small lesions by small reactions" and that it is not possible to generalize on the matter. It does seem evident that the tuberculin sensitivity produced by a vaccine containing a high proportion of dead bacilli and a small number of living ones is higher than would be expected, and there is some evidence that dead bacilli stimulate the tuberculin sensitivity produced by the living factor of the vaccine. 
From observations made by McKinstry in Jersey it appears that local reactions of under $4 \mathrm{~mm}$. diameter at the site of vaccination may not be accompanied by a positive tuberculin reaction, but that those over $5 \mathrm{~mm}$. in diameter are invariably tuberculin positive. Further observations are needed but it is probable that the post vaccination tuberculin test may, in the future, be dispensed with in all persons producing a local B.C.G. reaction of $5 \mathrm{~mm}$. or more. The degree of sensitivity will not necessarily be highest in those that show the severest local vaccination lesions. Palmer and Meyer (I95I) (23) have shown that the capacity to develop allergy after B.C.G. vaccination varies in children from different families. There is still no convincing explanation of the variation in ability to retain tuberculin sensitivity in different individuals who are not re-infected with virulent organisms. Such factors as diet, climate, race and concurrent infections may all play some part in bringing about reversion of the tuberculin reaction. In connection with this it is important to note that the haemagglutination reaction following B.C.G. vaccination shows that the antibody response is quantitatively very low and transient. It appears to bear no relation to cutaneous hypersensitivity to tuberculin.(24)

\section{ConcLusions.}

The one outstanding conclusion resulting from all the work that is being done on B.C.G. vaccination and tuberculin testing is that the problem is most complex and very confusing. There is little doubt that B.C.G. vaccination is harmless and that it enhances the individual's resistance against infection by virulent tubercle bacilli. The measurement of the degree of protection is extremely difficult and can only be done in humans by estimating the incidence of disease in those vaccinated and comparing it with that found in a comparable group of unvaccinated. This is a long and tedious investigation requiring considerable organization and skilled observation. To avoid this it is assumed that the degree of skin tuberculin sensitivity produced by vaccination is a measurement of the resistance produced. How far this assumption is justified and to what extent the tuberculin reaction indicates resistance to the infection, still requires careful study.

There is general agreement that tuberculin sensitivity is a protective phenomenon and for the present it is the only test we can readily apply to provide information, but its exact significance is still unknown. There is a great field for research in this direction and it is only by careful and continued observations in all parts of the World that the very important problem of the relationship between resistance and sensitivity will be solved. 


\section{REFERENCES.}

(1) Dubos, R. J., Schaefer, W. B., Pierce, C. H. (1953). Journ. Exp. Med. 97: 221 .

(2) Dubos, R. J., Schaefer, W. B., Pierce, C. H. (1953) ibid p. 189.

(3) Dubos, R. J.. Schaefer, W. B., Pierce, C. H. (1953) ibid p. 207.

(4) Hertzberg, G. (1949). Acta Tuberc. Scand. XXIII: 315.

(5) Wallgren, A. (1945). Nordisk. Med. $27: 35: 1715$.

(6) Ferguson, R. G. (1946). Amer. Rev. Tub. 54: 325.

(7) Aronson, J. D., Palmer, C. E. (1946). Pub. Health Rep. 61: 802.

(8) WILSON, G. S. (1947). B.M.J. ii : 855.

(9) Myers, J. A. (1952). Postgraduate Med. 12: Nos. 2, 3 and 5.

(10) Levine, M. I. (1952). Dis. of Chest. XXI: 5 : 513.

(11) IRVINE, K. N. I. (1949). B.C.G. Vaccination in Theory and Practice: Blackwell Scientific Publications, Oxford.

(12) van Deinse, F. (1951). Ann. Inst. Pasteur. 81: 126.

(13) Holm, J. (1946). Pub. Health Rep. 61: 1289.

(14) PAlmer, C. (1952). Lancet. i: 935.

(15) Dahlstrom, G., Difs, H. (1951). Acta Tuberc. Scand. 25: Suppl. 27.

(16) Cbron. W.H.O. (1950). 4: 331.

(17) Olsen, H. C. (1953). Acta Tuberc. Scand. XXVIII: 1-30.

(18) Wells, A. Q., Wylie, J. A. H. (1949). Lancet. i : 439.

(19) LeEs, A. W. (1951). Lancet. ii: 805.

(20) Proc. Roy. Soc. Med. (1952). 46: 253.

(21) Hertzberg, G. (1949). Acta Tuberc. Scand. XXIII: 338.

(22) Edwards, L. B., Palmer, C. E., Magnus, K. (1953). W.H.O. Monograph Series, Copenhagen.

(23) Palmer, C. E., Meyer, S. N. (1951). Pub. Health Rep. 66: 259.

(24) Haley, R. R., Davey, W. N., Adcock, J., Owen, C. R. (1952). Amer. Rev. Tuber. 66: 1: 58 . 